\title{
Understanding of hand muscles involvement: towards a linkage between biomechanical modeling and motor control theories
}

\author{
Franck Quaine ${ }^{1}$, Florent Paclet ${ }^{1,2}$, Laurent Vigouroux ${ }^{3}$ and François Moutet $^{4}$ \\ 1 GIPSA-Laboratory, CNRS UMR 5216, Control system department, SAIGA Team Grenoble University, France \\ 2 Department of Kinesiology The Pennsylvania State University University Park, 16802 PA, USA \\ 3 Institute of Mouvement Sciences, CNRS UMR 7287, Aix-Marseille Univ, Marseille, France \\ 4 Unit of Hand Surgery, CHU A. Michallon, Grenoble University, France
}

Received July 10th 2012 - Accepted November 28th 2012

\begin{abstract}
This study aimed at testing the pertinence of mimicking motor control theories in biomechanical models as a strategic direction for improving the understanding of muscle involvement. The theory of "minimization of the secondary moments of the hand" was added to an initial biomechanical model and was tested for several anatomic hand configurations (intact hand, paralyzed hand with radial nerve palsy, and transferred hand after tendon transfers). Results showed that the muscle sharing is governed by the minimisation of secondary moment for each anatomic hand configuration. In particular, co-contractions of extensor muscles were used to stabilize the wrist joint in both intact and transferred hands. Overall, this study showed that specific tuning of motor control theories rules may represent a strategic direction for improving biomechanical models. Enhancements are suggested in order to generalize this approach to others musculoskeletal systems.
\end{abstract}

Key words: Biomechanical model, optimization, hand/finger muscle, tendon transfers, motor control theories

Résumé. Utilisation des théories du contrôle moteur dans les modèles biomécaniques de la main pour mieux comprendre le recrutement musculaire.

L'objectif de ce travail est de montrer que les lois du contrôle moteur de la main peuvent être utilisées dans les modèles biomécaniques pour mieux comprendre le recrutement musculaire. Pour cela, le principe de «minimisation des moments secondaires » est implémenté dans un modèle biomécanique de la main en rajoutant l'équilibre de l'articulation du poignet dans la procédure d'estimation des tensions musculaires. Différentes conditions anatomiques de main sont testées : main intacte, main paralysée suite à une atteinte du nerf radial et main réanimée suite à un transfert tendineux (technique dite de «Tsugé »). Cela permet de modifier les capacités musculaires tout en gardant les moments secondaires identiques. Les résultats montrent que la distribution des forces entre les muscles des doigts et du poignet reste fidèle au principe de minimisation des moments secondaires quelque soit la condition anatomique testée. Lorsque le système musculaire le permet (main intacte et réanimée), le recours à l'utilisation de co-contractions avec les extenseurs des doigts reste la solution la plus avantageuse pour stabiliser les moments secondaires. Il apparaît que l'utilisation du principe de minimisation des moments secondaires permet d'améliorer les modèles biomécaniques dans leur capacité à montrer et expliquer les co-contractions. Au regard de ces résultats, la généralisation de cette méthodologie pour d'autres modèles biomécaniques est discutée.

Mots clés : Modèles biomécaniques, optimisation numérique, muscles de la main/doigts, transfert tendineux, théories du contrôle moteur

\section{Introduction}

The anatomy of the hand is one of the most complex human musculo-skeletal systems. The hand has 16 joints which include a total of 23 degrees of freedom (DoFs). Index (I), Middle (M), Ring (R) and Little (L) fingers present each 4 DoF. The Thumb (T) has 5 DoF while the wrist includes 2 DoF. To mobilize these DoF, 42 muscles categorized as intrinsic (origin in the palm of the hand) or extrinsic (origin located in the forearm) implies a high degree of muscular redundancy with more muscles than DoF (Chao, An, Cooney, \& Linscheid, 1989). As a consequence of this complexity, there is no simple solution that characterizes the muscle participation for 
a given movement. The understanding of the muscle involvement has been the subject of several studies in the last years and is of great importance for hand rehabilitation, finger surgery, neuroprostheses design and anthropomorphic robotics (Valero-Cuevas, Zajac, \& Burgar, 1998; Carrozza, Cappiello, Micera, Edin, Beccai, \& Cipriani, 2006).

Since direct measurement of all muscle forces are experimentally impossible, biomechanical models appears as a useful alternative to investigate muscle involvement. However hand modeling encounters with several technical, experimental and mathematical problems. One of the most important is the solving of the mathematical under-determinate problem associated with the muscle redundancy. The most advanced method to study muscles involvement combines biomechanical models with numerical optimization (Valero-Cuevas, et al., 1998; Gagnon, Lariviere, \& Loisek, 2001; Cholewicki \& McGill, 1994). This approach provides solutions that maximize or minimize an objective function related to physiological criterions such as the minimum of muscle tensions (Seireg \& Arvikar, 1973; Crowninshield \& Brand, 1981), and/or the minimum of muscle stress (Chao, et al., 1989; SanchoBru, Perez-Gonzalez, Vergata-Monedero, \& Guirantano, 2001; Paclet \& Quaine, 2012). For the hand, it has been demonstrated that introducing available intramuscular electromyography (EMG) of targeted extrinsic muscles as constraints in the optimization process improves the procedure (Vigouroux, Quaine, Labarre-Vila, Amarantini, \& Moutet, 2007). Unfortunately, this method remains unexploited and it is very unlikely that it will be used in routine for the study of finger muscle recruitment. Moreover, even if muscle forces are estimated with this method, the selection of a particular muscle sharing by the central nervous system (CNS) remains unexplained. Co-contractions with the extensor muscles observed during a pure flexion task are an example.

In this study, we propose a linkage between two major hand research fields: biomechanical modeling and hand/finger control. We think that mimicking appropriately the fine tuning of specific motor control theories rules in the model design would provide more realistic solutions and could give explanations concerning the muscle involvement, particularly for co-contractions. One relevant theory of hand/finger control concerns the force sharing among fingers presented as a model of the redundancy problem ( $\mathrm{Li}$, Latash \& Zatsiorsky, 1998; Vigouroux, Ferry, Colloud, Paclet, Cahouet, \& Quaine, 2008). This theory affirms that the CNS shares the force among fingers in order to minimize the wrist joint stabilization moments (i.e., secondary moments). Paclet and Quaine (2012) have adapted this theory for finger biomechanical models by including the wrist mechanical equilibrium in the same computation process than the fingers. With this procedure, they showed that muscle involvements were different than those observed classically. Particularly, the muscle sharing was characterized by co-contractions between flexor and extensor muscles.
In the current study, we aim to test the robustness of this approach with the investigation of different hand anatomical configurations. The example of tendon transfer surgery is particularly relevant to test the procedure since some reversals of functions were observed in the transferred muscles (Leffert \& Meister, 1976; Illert, Trauner, Weller, \& Wiedemann, 1986). Thus, the rerouting of some tendons will affect the muscle involvement for joint equilibrium, while the mechanical constraints of the hand remain unchanged (i.e., secondary moments of the wrist). We hypothesized that if the CNS shares the muscle forces according to the minimization of the secondary moments, the finger muscles restored after the transfer should specifically participate to the wrist equilibrium, in particular with co-contractions.

We proposed three versions of a hand biomechanical model including the intact, the paralyzed and the specific mapping of the transplanted muscles. Input data were collected from one volunteer patient with radial nerve palsy throughout his care pathway, before and after tendon transfers. Muscle force sharing was then compared for the intact, the paralyzed and the transferred hand.

\section{Material and methods}

\subsection{Subject}

One patient (male, 26 years old) participated in the study. He benefited from a tendon transfer surgery after a traumatic radial nerve palsy affecting his capacity for extension functions of his right hand.

The Tsugé's procedure (Tsugé \& Adashi, 1969) was used. It corresponds to the transfer of the pronator teres to the extensor carpi radialis brevis, the flexor carpi radialis to the extensor digitorum communis, the palmaris longus to the extensor pollicis brevis and the $4^{\text {th }}$ flexor digitorum superficialis to the extensor pollicis longus (Tab. 1).

This experimentation was approved by the consultative committee for the protection of persons in biomedical research (CCP, Grenoble, France). Data corresponding to the intact hand were obtained from Paclet and Quaine (2012).

\subsection{Experimental device and procedure}

The subject was seated with the right arm placed in the force measurement device. The shoulder was abducted at $45^{\circ}$ and flexed at $0^{\circ}$. The elbow flexed at $90^{\circ}$ and the wrist extended at $30^{\circ}$ with the forearm horizontal (Fig. 1).

Each finger was placed in a separate ring connected with a tri-axial load cell (3D Force sensors, model 9017B, Kistler, Switzerland). The distance between each load cell was $26 \mathrm{~mm}$ along the medio-lateral axis. The ring was located at the half the length of the distal phalanx. The 
Table 1. A glossary of abbreviations used for the analysis of hand musculature. $\mathbf{X}$ indicates a paralyzed muscle. Bold italic muscle indicates a muscle used as a donor for the surgery. PT indicates the Pronator Teres muscle. $f$ indicates the $f$ th finger $(f=\mathrm{I}, \mathrm{M}, \mathrm{R}$, and $\mathrm{L})$.

\begin{tabular}{|c|c|c|c|}
\hline Muscles & Intact hand & Paralyzed hand & Transferred hand \\
\hline Extensor carpi radialis brevis & ECRB & $\boldsymbol{X}$ & $P T$ \\
\hline Extensor carpi radialis longus & ECRL & $\boldsymbol{x}$ & $\boldsymbol{x}$ \\
\hline Extensor carpi ulnaris & $\mathrm{ECU}$ & $\boldsymbol{X}$ & $\boldsymbol{x}$ \\
\hline Extensor digitorum communis & $\mathrm{EDC} f$ & $\boldsymbol{x}$ & $F C R f$ \\
\hline Extensor digitorum indicis & EDI & $\boldsymbol{x}$ & $\boldsymbol{x}$ \\
\hline Extensor digitorum minimi & EDM & $\boldsymbol{x}$ & $\boldsymbol{x}$ \\
\hline Extensor pollicis brevis & $\mathrm{EPB}$ & $\mathbf{x}$ & $P L$ \\
\hline Extensor pollicis longus & EPL & $\mathbf{x}$ & $4^{\text {th }} F D S$ \\
\hline Flexor carpi radialis & FCR & FCR & $\boldsymbol{x}$ \\
\hline Flexor carpi ulnaris & FCU & FCU & FCU \\
\hline Flexor digitorum profundus & $\operatorname{FDP} f$ & $\operatorname{FDP} f$ & FDP \\
\hline Flexor digitorum minimi & FDM & FDM & FDM \\
\hline Flexor digitorum superficialis & FDS $f$ & $\operatorname{FDS} f$ & $\operatorname{FDS} f\left(\boldsymbol{X} 4^{\text {th }} F D S\right)$ \\
\hline Flexor pollicis longus & FPL & FPL & FPL \\
\hline Adductor pollicis longus & APL & $\boldsymbol{x}$ & $\mathbf{x}$ \\
\hline Lumbricales & $\operatorname{LUf}$ & LUf & $\mathrm{LU}$ \\
\hline Palmaris longus & $\mathrm{PL}$ & PL & $x$ \\
\hline Radial interosseus & $\mathrm{RI} f$ & $\mathrm{RI} f$ & RI \\
\hline Ulnar interosseus & $\mathrm{UI} f$ & $\mathrm{UI} f$ & UI \\
\hline
\end{tabular}

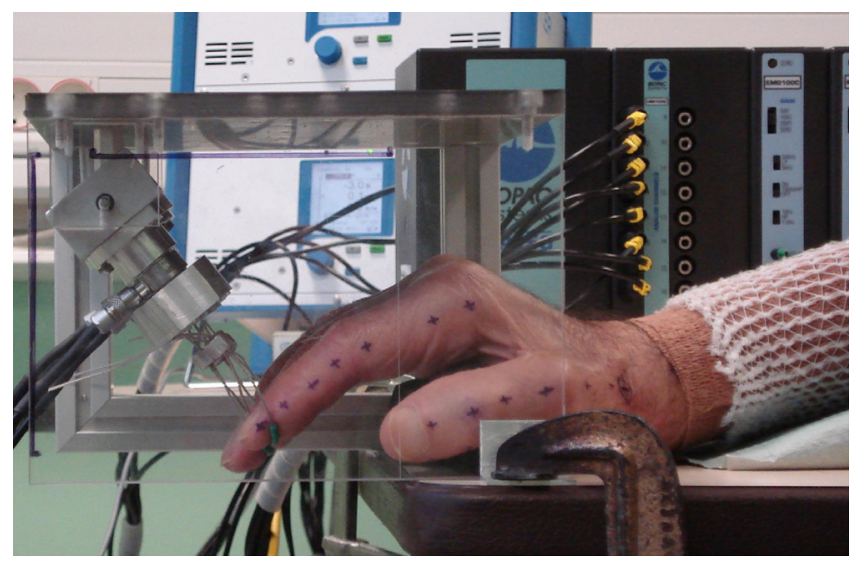

Fig. 1. Experimental setup. Each finger was placed in a separate ring connected with a 3 D Force sensor. Anatomical landmarks were located proximally and distally in order to reflect the longitudinal axis of each segment. The subject was asked to press downwards.

thumb did not exert any supplementary force on the support (visually check at each trial by the experimenter). An iron cable was used to connect the finger with the force sensor. Cables with different length and angle were used to compensate for the different finger sizes.

The wrist and the index finger joint angles were identified in the sagittal plane and were assumed to be similar for the others fingers. Anatomical landmarks were located proximally and distally in order to reflect the longitudinal axis of each segment. A digital camera (Sony, DSC-W40) recorded the coordinates of these points during force application. The angle between the distal segment and the cable was measured for each finger.

The subject was asked to apply a maximal force downwards (Valero-Cuevas, et al., 1998) using the four fingers. After warm up, the patient performed three trials, 1 month before and 6 months after surgery.

\subsection{Hand biomechanical model}

The model was constituted from Paclet and Quaine (2012), Vigouroux, et al., (2007) and Vigouroux, Quaine, Labarre-Vila, \& Moutet (2005) and was adapted sequentially to the paralyzed and transferred hand. It includes the four long fingers joints plus the wrist joint. The thumb was not included in the model since this finger did not apply any external force during our experiment. However, since the extrinsic muscles of the thumb create great moments at he wrist joint, they were inserted in the biomechanical model as proposed by Paclet and Quaine (2012). Following our hypothesis concerning the wrist equilibrium and the secondary moments of the fingers, the wrist was included in the same computing process of the model.

Hence, the set of equations in the model provides 37 unknown tendon tensions for 18 DoF. 
The extensor mechanism of the fingers was modeled as in Vigouroux, et al. (2007) and An, Chao, Cooney \& Linscheid (1979). It introduces $\beta$ coefficient for EDC, LU, UI and RI muscles for each finger in the optimization process. The force fraction transmitted by muscle tendons to the extensor mechanism bands were obtained from Eyler and Markee (1954).

The segment lengths and moment arms were obtained from the anthropometric tables of Buchholz (1992), An, et al. (1979), Lemay and Crago (1996); Brand \& Hollister (1999).

The model runs from the distal point of contact of the external force (i.e., the ring finger contact) of each finger until the wrist joint. The static moment equilibrium equation states that the external force moments at the joints are counterbalanced by moments produced by tendon tensions and ligaments as:

$$
[R] \cdot\{T\}+\{L\}+\{F\}=\{0\}
$$

where the matrix $[R]$ is a $18 \times 37$ matrix containing moment arms of the 37 muscles for the $18 \mathrm{DoF} ;\{T\}$ is a vector containing the 37 unknown muscle tensions; $\{L\}$ is a vector containing the passive moments over the distal and metacarpophalangeal joints due to the ligament and passive joint structures and $\{F\}$ is a vector representing the moments of external force at the 18 DoF.

\subsection{The optimization process}

The min-max optimization criterion was used to solve the under-determinate problem (Rasmussen, Damsgaard, \& Voigt, 2001). This criterion assumes that the load sharing between the muscles follows the way that the maximum muscle stress given by the ratio $t i / P C S A i$ for $i$ th muscle (where PCSA $i$ is the physiological cross sectional area of the $i$ muscle) is minimized. In other words, this criterion gives the solution which minimizes the maximal muscle stress value among the finger muscles and hence tends to distribute evenly the load between the muscles.

Find $t i$ and associated $\beta$ coefficients that minimize:

$$
f\left(t_{i}\right)=\max \left(\frac{t_{i}}{P C S A_{i}}\right)
$$

With $t i(i=\operatorname{FDP} f, \operatorname{FDS} f$, EDC $f$, LU $f$, UI $f$, RI $f$, EDI, EDM, EPB, EPL, FCR, FCU, FDM, FPL, APL, PL, ECRB, ECRL and ECU) and the extensor mechanism coefficients $\beta$ EDC $f, \beta \mathrm{LU} f, \beta \mathrm{UI} f$ and $\beta \mathrm{RI} f$; where $f$ is corresponding to the $f$ th finger $(f=\mathrm{I}, \mathrm{M}, \mathrm{R}$, and $\mathrm{L})$. PCSAi were obtained in Chao, et al. (1989).

We introduce the following constraints: $0 \leqslant t i$ and ti must be superior or equal to zero and subject to the equilibrium constraints expressed in Equation (1) and in the extensor mechanism model.

The min-max criterion is known to be rather sensitive to the selection of the starting point of optimization algorithm. In order to avoid side effects and without any useful additional information in the literature, we have chosen the half of the maximal theoretical forces for each muscle (i.e., $t_{i \max }=P C S A_{i} \times \sigma_{\max }$ where $\sigma_{\max }$ is the muscle stress fixed to 35 N.cm ${ }^{2}$ in Valero-Cuevas, et al., (1998)) as starting point. All computations were performed with the MATLAB optimization toolbox (The Math Works, Inc., USA).

The model was adapted for three different hand configurations. The first corresponds to an intact hand with no anatomical restriction based on Paclet \& Quaine (2012). The second corresponds to the paralyzed musculature of the patient with radial nerve palsy. In this model we have removed the extensor paralyzed muscles. As a consequence, no co-contraction was possible with this model. Finally, the third configuration includes the transferred tendons after the surgery according to the Tsugé procedure. Without any data in the literature concerning the force sharing pattern for finger extensor force execution in the FCR muscle after its transfer, we have shared the PCSA among the fingers according to Quaine, Paclet, Letue and Moutet (2011). The force generation capabilities in the restored finger extensors were $32 \%, 27 \%$, 22 and $18 \%$ for I, M, L, and R, respectively. Since tendon moment arms were not modified with the tendon transfer, the respective moment arms in the transferred hand model were equal to those of intact muscles.

\subsection{Analysis}

Both applications of the model for the paralyzed hand and the transferred hand work with input data recorded during specific experiments performed with the patient before and after the surgery. Three trials were performed and averaged for separated external finger forces and joint angles. Tendon tensions were computed and comparisons were made between the intact versus both paralyzed and transferred hands.

For the intact hand, data were obtained in Paclet and Quaine (2012). In this study, the external force magnitude used was low compared to the current values. In order to avoid magnitude effect, we have used the normalization process given in Valero-Cuevas, et al. (1998). Each tendon tension was divided by the external force magnitude applied at the associated finger.

\section{Results}

\subsection{External forces and finger joint angles}

Input data are presented in Table 2. For the intact hand, the total force given in Paclet and Quaine (2012) amounted to $38 \mathrm{~N}$ and was shared among the fingers as $31 \%, 42 \%, 18 \%$ and $8 \%$ for I, M, R, L respectively. For the paralyzed hand, the total force was $62 \mathrm{~N}$ with $13 \%$, $49 \%, 16 \%$ and $21 \%$ for I, M, R and L; whereas in the transferred hand, the force amounted to $113 \mathrm{~N}$ and was 
Table 2. Input data. For the intact hand, data were obtained in Paclet and Quaine (2012). For the paralyzed and transferred hand, they were collected with one patient before and after tendon transfer surgery. RC corresponds to the wrist joint.

\begin{tabular}{ccccc}
\hline & & Intact hand & Paralyzed hand & Transferred hand \\
\hline \multirow{2}{*}{ External force } & $\mathrm{I}$ & 12 & 18 & 8 \\
$(\mathrm{~N})$ & $\mathrm{M}$ & 16 & 36 & 30 \\
& $\mathrm{R}$ & 7 & 32 & 9 \\
& $\mathrm{~L}$ & 3 & 26 & 13 \\
\hline \multirow{3}{*}{ Joint angle } & $\mathrm{DIP}$ & 24 & 20 & 18 \\
$\left(^{\circ}\right)$ & $\mathrm{PIP}$ & 23 & 22 & 20 \\
& $\mathrm{MCP}$ & 21 & 18 & 20 \\
& $\mathrm{RC}$ & -27 & -15 & -14 \\
\hline
\end{tabular}

shared as $16 \%, 32 \%, 28 \%$ and $23 \%$ for each finger. The joint angles were slightly different but remain in a similar range.

\subsection{Muscle tendon tensions}

Tendons tensions are expressed in percentage of the external force applied by each finger (Fig. 2). For the paralyzed hand, the extensor muscles were removed from the model, so their tensions were set to zero. For the transferred hand, the paralyzed muscles were replaced by the donor muscles, as presented in Table 1.

Results showed that the tensions in the muscles mobilizing the fingers are modified for each hand anatomical configuration and are different for each finger. In particular, the $\mathrm{R}$ and $\mathrm{L}$ fingers are highly modified by these factors whereas the $\mathrm{M}$ finger presents limited variations in each case.

The comparison between intact versus paralyzed hand shows differences mainly localized for the flexor muscles. These differences depend upon the finger considered. For I and $\mathrm{M}$ fingers, FDP and FDS muscles twice reduce their involvement in the paralyzed hand (from 2.5 to 1.4 times the external force), while the involvement of the other muscles remain unchanged (this is particularly true for the $\mathrm{M}$ finger). For $\mathrm{R}$ and $\mathrm{L}$ fingers, the major reductions concern the FDP and the UI muscles, while FDS muscle does not exhibit change. In the $\mathrm{R}$ finger, FDP and UI ratios decrease from 4.8 to 1.9 and 5.3 to 2.3 , respectively. At the wrist joint, the FCU is two times reduced, while all the others active muscles remain unchanged.

The comparison between intact versus transferred hand illustrates how the model adapts to the tendon transfers. In the transferred hand the paralyzed muscles were substituted by the donor muscles which were set at zero tension. Important changes were noted for the FCR muscle (neo-extensor muscle) and FDP and FDS for each finger. For I and M fingers, FDP and FDS regained their initial involvement observed in the intact hand. In $\mathrm{R}$ finger, the removal of the FDS is replaced by the larger increase in the FDP involvement. The latter increased until 6.5 times the external force, whereas both flexors were evenly involved for the $\mathrm{R}$ finger (between 2 and 3 times the external force for FDP and FDS, respectively).

It is noticeable to observe that all the neo-extensor muscles (FCR $f$ ) for each finger present a growth in contribution, having a minimum for I finger at 0.7 times the external force and a maximum of 4.1 times the external force for the $\mathrm{R}$ finger.

At the wrist, both FCR and PL muscle tensions were set to zero since both were used as donor muscles. The loss of the FCR was filled by the FCU which recovered its initial involvement level as in the intact hand. PT muscle used in substitution of the wrist extensor (ECRB) did not act, whereas the FPL largely increased its participation from 2 to 5.5 times the external force.

\subsection{Moments applied on the transversal section of the wrist}

Computed moments at the wrist are presented in Figure 3. Both wrist and finger muscles act in flexion to balance the extension moment of the external force, while wrist and finger muscles balance each other in radial and ulnar deviation.

The flexion moment was due to the wrist and finger muscles (68 and 32\%, respectively) for the intact hand. For the paralyzed hand, wrist and finger muscles represent $72 \%$ and $28 \%$ of the resultant flexion moment. For the transferred hand, this repartition increases to $80 \%$ for the wrist moment and $20 \%$ for the finger moment. For both the paralyzed and transferred hands, the radial deviation created by the wrist muscle moment was counterbalanced by the ulnar moment of the finger muscles $(78 \mathrm{Ncm}$ in the paralyzed hand and $110 \mathrm{Ncm}$ in the transferred hand).

\section{Discussion}

This study aimed at testing the pertinence of using motor control theories as a strategic direction for improving biomechanical models. We have chosen to mimic the principle of minimization of the secondary moments of the 

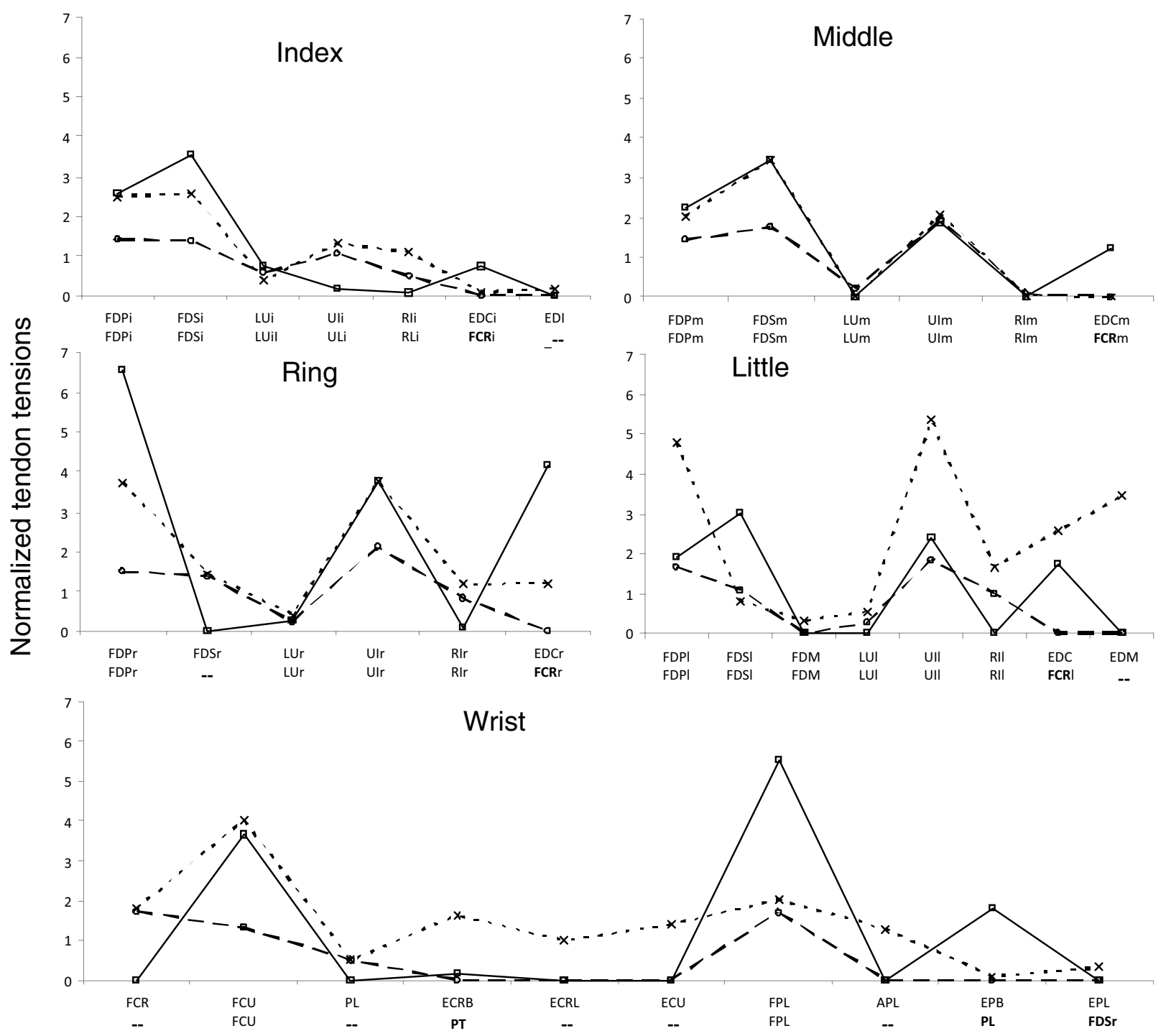

Fig. 2. Tendon tension estimations. Tensions are expressed in percentage of the external force. On the abscissa axis, the first line corresponds to the intact hand, the second to the transferred hand. Bold muscle corresponds to the transferred muscle, corresponds to a paralyzed muscle. $x$ intact hand, o paralyzed hand, $\square$ transferred hand.

hand at the level of tendon tensions. In order to reproduce this principle, the wrist joint equilibrium was added to a biomechanical model of the finger for pressing tasks. Tendon transfers surgery was selected for testing this approach since transferred muscles have reversal functions while the mechanical constraints of the hand remain unchanged (i.e., secondary moments). We proposed a model adapted sequentially for an intact, a paralyzed (i.e., radial nerve palsy) and a transferred hand. In the paralyzed hand, the palsied extensor muscle tensions were set at zero. In the restored hand, the palsied muscles were substituted by several active muscles according to the rerouting obtained in Tsugé and Adashi (1969).

Our results confirmed previous results given for the intact hand in Paclet and Quaine (2012). Both wrist and finger muscles were synergistic to balance the wrist in flexion, but antagonist to balance the joint in radial-ulnar direction. The wrist muscle resultant moment acted in radial deviation while the finger muscle moment acted in ulnar deviation. In the intact hand, co-contractions with the extensor muscles in the ring and little finger have been presented as the most appropriated solution to obtain this equilibrium. In the paralyzed hand, this muscle force sharing is not feasible since extensor muscles were inactive. The adapted model includes this disability and displays one other alternative solution. The geometrical repartition of the muscle tendons at the wrist joint illustrates this solution. Figure 4a shows a muscle dissymmetry in the radial-ulnar direction since the FCU remains the single wrist muscle to the ulnar side. As a consequence, in addition to create flexion moment, finger flexor muscles balance the wrist with the constraint 

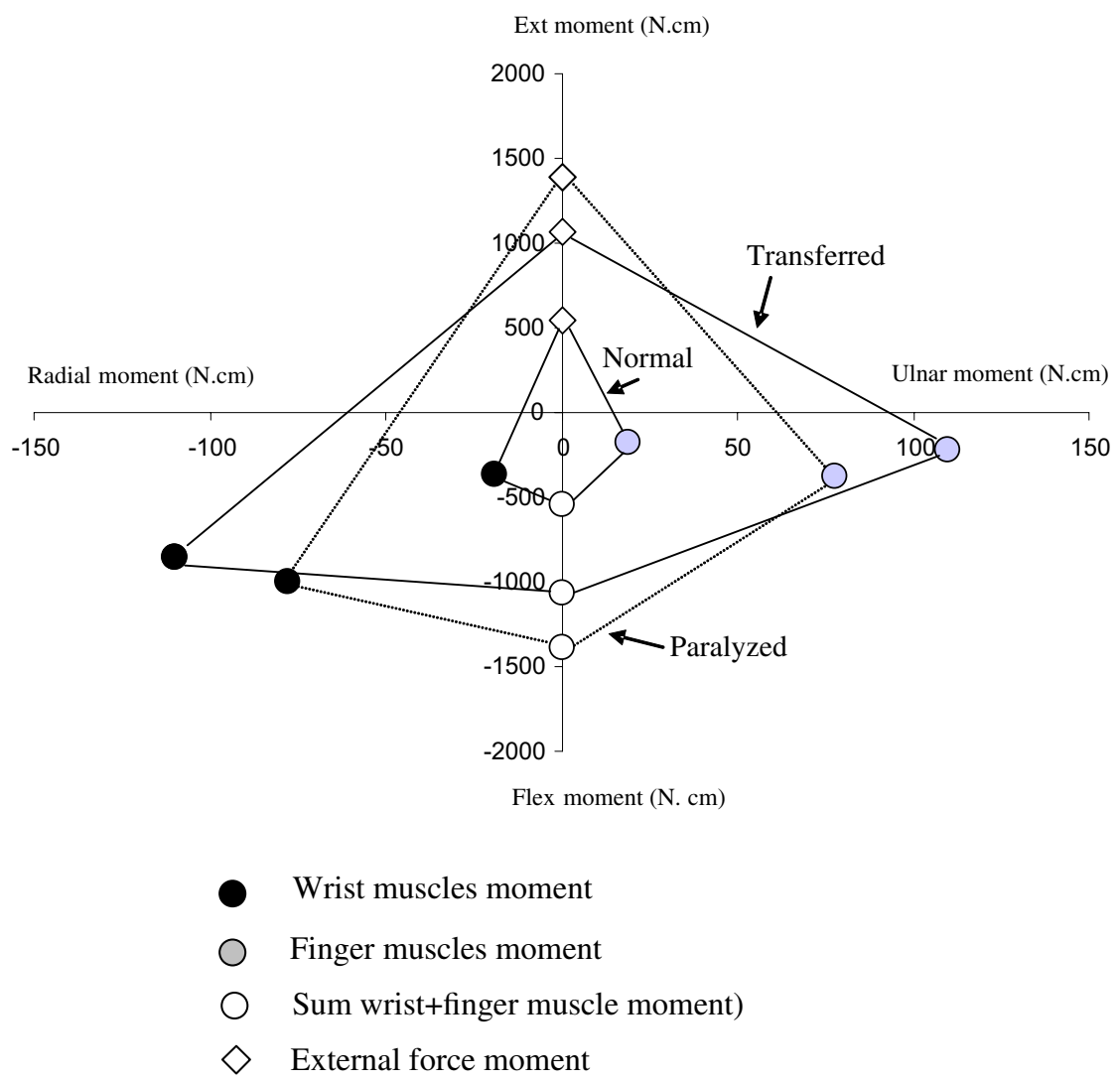

Fig. 3. Resultant moments. Flexion/extension and radial/ulnar moments represented at the wrist transversal section for the intact, paralyzed and transferred hand.

to create ulnar moment. This moment reduces the FCU moment and thus facilitates its action for counterbalancing PL and FCR moments. This result is in line with the principle of minimization of the secondary moments. Hence, in the paralyzed hand, we observe that the wrist and finger muscle moments remain still distinctly separated in radial and ulnar deviation. We conclude that the finger muscle moments participate always to balance the wrist in ulnar deviation despite the absence of extensor muscles. This observation is novel and highlights the mechanical constraints of the paralyzed hand.

Conversely, in the transferred hand, the re-routing of tendons display available reconstructed co-contractions (Fig. 4b) with the PT and FCR re-routed muscles. These transfers modify the muscle force sharing pattern since they impacted the flexion but also the radial-ulnar balance requirements. To overcome these constraints, the co-contraction increases in the finger transferred muscle (i.e., FCR) and decreases in the wrist transferred muscle (i.e., $\mathrm{PT}$ ). These actions bring a reduction in FCU muscle moment and balance the wrist in radial-ulnar direction. This confirms the reversal functions given by Leffert and Meister (1976) and Illert et al., (1986) and helps to understand the mechanisms underlying these changes. An important feature is that the provided estimations preserve the division in the wrist and finger muscle actions in radial and ulnar deviation, respectively. This result is in accordance with the principle of minimization of secondary moments: based on these results, it can be hypothesized that the CNS associates muscle functions with different hand anatomical configurations in order to minimize the secondary moments. We show that the suppression of the flexor muscles for the transfers does not deteriorate the flexion function but rather induces motor command reorganization for the flexor muscles used as extensor accordingly to the minimization of the secondary moments. The growth in the relative part in flexion moment for the wrist muscles from $68 \%$ in the intact hand to $80 \%$ in the transferred hand shows that the relative wrist muscle moments increase when redundancy decreases. This result agrees well with the concept of muscular abundance proposed by Latash (2000) and Karol, et al. (2011). It is being hypothesized that the current results point toward the principle of minimization of secondary moment is inversely proportional to the level of motor redundancy. One explanation is probably that releasing multi joint muscles for the transfers complicates the optimization to find optimal solution and constrains the procedure to solve the redundancy problem at each joint sequentially. Future research should examine more in details the link between motor redundancy and motor abundance. For example, instead of reducing 


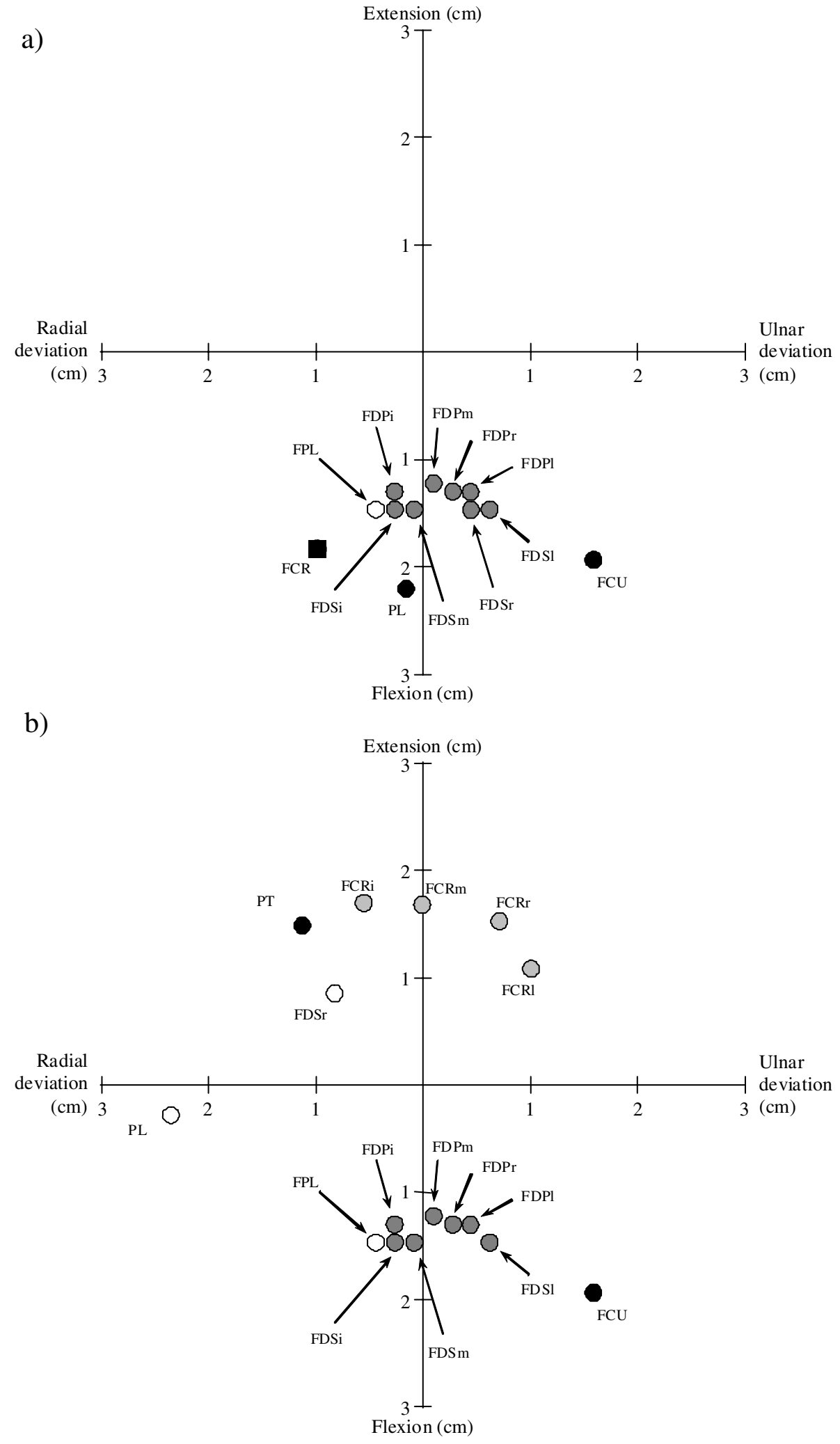

Fig. 4. Muscle anatomical features. Comparison of muscle moment arms between the paralyzed hand (a) and the transferred hand (b) at the wrist transversal section. 
hand actuators, it should be relevant to restrict DoFs as in carpectomy or four-corner fusion for osteoarthrosis treatment (Bisneto, Freitas, Leomil de Paula, Mattar, \& Zumiotti, 2011).

This work shows that the wrist equilibrium requirement, and particularly the secondary moment, gives explanation for co-contractions observed in finger extensor muscles. This point is of importance as it explains the activation of extensor muscles previously observed by EMG (Vigouroux, et al. 2007) for finger flexion tasks and it attributes one explanation in terms of muscle tensions to the minimization of secondary moment (Li, et al. 1998).

Some limitations should be considered for our study: first, the model would be improved by the inclusion of the entire thumb column with its own intrinsic muscles for grasping tasks. Second, the different levels of co-contraction observed for each finger in FCR does not agree with the unique compartment of this muscle in contrast with the separated EDC compartments (Leijnse, Carter, Gupa, \& McCabe, 2008). One explanation lies probably in the specific tendon tensioning performed by the surgeon for the different fingers. However, before conclusions can be drawn, future experiments focusing on this point should be planned.

Overall our results showed that the muscle involvement in the paralysed and transferred hand, although different from the intact hand, is governed by the same rules (i.e., minimisation of secondary moment). At this stage, it is relevant to observed that this result is only available when adding wrist joint equilibrium in the finger model. This confirms the relevance to consider the hand motor control theories as a strategic manner to improve models. However, in the current study, we only adapt the principle of minimizing the secondary moments by adding balance of the wrist in the model. Further improvement would be to express this principle in term of additional equality or inequality constraints added to the procedure of optimization. In order to achieve this goal, additional well known motor control principles, such as the "enslaving" and/or the "force deficit" (Zatsiorsky, Li, \& Latash, 2000; Martin, Zatsiorsky, \& Latash, 2011; Zatsiorsky, Gregory, \& Latash, 2002) should be tested. This would probably permit to characterize peripheral and central constraints on performance (Schieber \& Santello, 2004). Moreover, if finger muscle involvement obeyed these laws, it should be hypothesized that other musculo-skeletal systems should be governed on the same criteria. Therefore, adapting this approach to other several musculo-skeletal systems, different from the hand, might be relevant to understand the muscles involvement.

In conclusion, this work draws appropriately on motor control theories rules as a strategic direction for improving biomechanical models with optimization. Including the wrist joint in a hand biomechanical model mimics the principle of minimization of secondary moments. It allows characterizing the muscle involvement in an intact, paralyzed and transferred hand. A specific radial-ulnar partition (i.e., secondary moment) between the wrist and finger muscles is proposed. At the wrist joint, this partition gives rationale for co-contractions with finger extensor muscles, which represents a relevant improvement for finger biomechanical models. For generalization, one reflexion should be engaged to enunciate the principle of minimization of secondary moments in terms of optimization criteria.

\section{Bibliography}

An, K., Chao, E., Cooney, W., \& Linscheid, R. (1979). Normative model of human hand for biomechanical analysis. Journal of Biomechanics, 12, 775-788.

Bisneto, E., Freitas, M., Leomil de Paula, E., Mattar, R., \& Zumiotti, A. (2011). Comparison between proximal row carpectomy and four-corner fusion for treating osteoarthrosis following carpal trauma: a prospective randomized study. Clinics, 66, 51-55.

Brand, P., \& Hollister, A., (1999). Clinical Mechanics of the Hand. Saint Louis : 3rd edition Mosby.

Buchholz, B., (1992). Anthropometric data for describing the kinematics of the human hand. Ergonomics, 35: 261-273.

Carrozza, M., Cappiello, G., Micera, S., Edin, B., Beccai, L., \& Cipriani, C. (2006). Design of a cybernetic hand for perception and action. Biological Cybernetic, 9, 629-644.

Chao, E., An, K., Cooney, W., \& Linscheid, R.L. (1989). Biomechanics of the Hand. Singapore: World Scientific.

Cholewicki, J., \& McGill, S. (1994). EMG assisted optimization: a hybrid approach for estimating muscle forces in an indeterminate biomechanical model. Journal of Biomechanics, 27, 1287-1289.

Crowninschield, R., \& Brand, R. (1981). A physiologically based criterion of muscle force prediction in locomotion. Journal of Biomechanics, 14, 793-801.

Eyler, D., \& Markee, J. (1954). The anatomy and function of the intrinsic musculature of the fingers. Journal of Bone and Joint Surgery, 36, 1-18.

Gagnon, D., Lariviere, C., \& Loisek, P. (2001). Comparative ability of EMG, optimization, and hybrid modelling approaches to predict trunk muscle forces and lumbar spine loading during dynamic sagittal plane lifting. Clinical Biomechanics, 16, 359-372.

Illert, M., Trauner, M., Weller, E., \& Wiedemann, E. (1986). Forearm muscles of man can reverse their function after tendon transfers: an electromyographic study. Neuroscience Letters, 67, 129-134.

Karol, S., Kim, Y., Huang, J., Kim, Y., Koh, K., Yoon, B., \& Shim, J. (2011). Multi-finger pressing synergies change with the level of extra degrees of freedom. Experimental Brain Research, 208, 359-367.

Latash, M. (2000). There is no motor redundancy in human movements. There is motor abundance. Motor Control, 4, 259-260.

Leffert, R., \& Meister, M. (1976). Patterns of neuromuscular activity following tendon transfer in the upper limb: a preliminary study. Journal of hand Surgery, 3, 181-189. 
Leijnse, J., Carter, S., Gupa, A., McCabe, S. (2008). Anatomic basis for individual surface EMG and homogeneous electrostimulation with neuroprostheses of the extensor digitorum communis. Journal of Neurophysiology, 100, 64-75.

Lemay, A., \& Crago, E. (1996). A dynamic model for simulating movements of the elbow, forearm and wrist. Journal of Biomechanics, 29, 1319-1330.

Li, Z., Latash, M., \& Zatsiorsky, V. (1998). Force sharing among fingers as a model of the redundancy problem. Experimental Brain Research, 119, 276-286.

Martin, J., Zatsiorsky, V., \& Latash, M. (2011). Multi-finger interaction during involuntary and voluntary single finger force changes. Experimental Brain Research, 208, 423-435.

Quaine, F., Paclet, F., Letué, F., \& Moutet, F. (2012). Force sharing and neutral line during finger extension tasks. Human Movement Science, 31, 749-757.

Paclet, F., \& Quaine, F. (2012). Motor control theories improve biomechanical model of the hand for finger pressing tasks. Journal of Biomechanics, 45, 1246-1251.

Rasmussen, J., Damsgaard, M., \& Voigt, M. (2001). Muscle recruitment by the $\min / \max$ criterion-a comparative numerical study. Journal of Biomechanics, 34, 409-415.

Sancho-Bru, J., Perez-Gonzalez, A., Vergara-Monedero, M., \& Giurintano, D. (2001). A 3-D dynamic model of human finger for studying free movements. Journal of Biomechanics, 34, 1491-500.

Schieber, M., \& Santello, M. (2004). Hand function: peripheral and central constraints on performance. Journal of Applied Physiology, 96, 2293-2300.
Seireg, A., \& Arvikar, R. (1973). A mathematical model for evaluation of force in lower extremities of the musculoskeletal system. Journal of Biomechanics, 6, 313-326.

Tsugé, K., \& Adachi, N. (1969). tendon transfer for extensor palsy of forearm. Hiroshima Journal of Medicine Sciences, 18, 219-232.

Valero-Cuevas, F., Zajac, F., \& Burgar, C. (1998). Large index-fingertip forces are produced by subjectindependent patterns of muscle excitation. Journal of Biomechanics, 31, 693-703.

Vigouroux, L., Quaine, F., Labarre-Vila, A., \& Moutet, F. (2005). Estimation of finger muscle tendon tensions and pulley forces during specific sport climbing grip techniques. Journal of Biomechanics, 40, 2846-2856.

Vigouroux, L., Quaine, F., Labarre-Vila, A., Amarantini, D., \& Moutet, F. (2007). Using EMG data to constrain optimization procedure improves finger tendon tension estimations during static fingertip force production. Journal of Biomechanics, 40, 2846-2856.

Vigouroux, L., Ferry, M., Colloud, F., Paclet, F., Cahouet, V., \& Quaine, F. (2008). Is the principle of minimization of secondary moment validated during various fingertip force production conditions? Human Movement Science, 27, 396-407.

Zatsiorsky, V., Li, Z., \& Latash, M. (2000). Enslaving effects in multi-finger force production. Experimental Brain Research, 131, 187-195.

Zatsiorsky, V., Gregory, R., Latash, M. (2002). Force and torque production in static multifinger prehension: biomechanics and control. II. Control in Biological Cybernetic, $87,40-49$. 